\title{
Workspace, Joint space and Singularities of a family of Delta-Like Robot
}

\author{
Ranjan Jha ${ }^{\mathrm{a}}$, Damien Chablat ${ }^{\mathrm{b}, *}$, Luc Baron ${ }^{\mathrm{c}}$, Fabrice Rouillier ${ }^{\mathrm{d}}$, Guillaume \\ Moroz $^{\mathrm{e}}$ \\ ${ }^{a}$ BioMedical Instrumentation Division, CSIR-Central Scientific Instruments Organisation, \\ Chandigarh, India \\ ${ }^{b}$ Laboratoire des Sciences du Numérique de Nantes, UMR CNRS 6004, Nantes, France. \\ ${ }^{c}$ Department of Mechanical Engineering, École Polytechnique de Montréal, Québec, Canada \\ ${ }^{d}$ INRIA Paris-Rocquencourt, Institut de Mathématiques de Jussieu, UMR CNRS 7586, \\ Paris, France. \\ ${ }^{e}$ INRIA Nancy-Grand Est, Nancy, France.
}

\begin{abstract}
This paper presents the workspace, the joint space and the singularities of a family of delta-like parallel robots by using algebraic tools. The different functions of SIROPA library are introduced, which is used to induce an estimation about the complexity in representing the singularities in the workspace and the joint space. A Gröbner based elimination is used to compute the singularities of the manipulator and a Cylindrical Algebraic Decomposition algorithm is used to study the workspace and the joint space. From these algebraic objects, we propose some certified three-dimensional plotting describing the shape of workspace and of the joint space which will help the engineers or researchers to decide the most suited configuration of the manipulator they should use for a given task. Also, the different parameters associated with the complexity of the serial and parallel singularities are tabulated, which further enhance the selection of the different configuration of the manipulator by comparing the complexity of the singularity equations.
\end{abstract}

Keywords: Delta-like robot, Cylindrical algebraic decomposition, Workspace, Gröbner basis, Parallel robot, Singularities

\footnotetext{
*. Corresponding author

Email addresses: Ranjan.Jha@csio.res.in (Ranjan Jha), Damien.Chablat@cnrs.fr (Damien Chablat), Luc.Baron@polymtl.ca (Luc Baron), Fabrice.Rouillier@inria.fr (Fabrice Rouillier), Guillaume.Moroz@inria.fr (Guillaume Moroz)
} 


\section{Nomenclature}

$\begin{array}{ll}\text { SIROPA } & \text { Library for manipulator singularities analysis } \\ \text { CAD } & \text { Cylindrical Algebraic Decomposition } \\ \text { IKP } & \text { Inverse Kinematics problem } \\ \text { DKP } & \text { Direct Kinematics problem } \\ \text { det } & \text { Determinant of Jacobian matrix } \\ \text { R } & \text { Revolute Joint } \\ \text { P } & \text { Prismatic Joint } \\ \text { S } & \text { Spherical Joint } \\ \rho & \text { Actuated Joint Variables } \\ \text { X } & \text { Pose Variables } \\ \text { A } & \text { Direct parallel Jacobian matrices } \\ \text { B } & \text { Inverse serial Jacobian matrices }\end{array}$

\section{Introduction}

The workspace can be defined as the volume of space or the complete set 5 of poses which the end-effector of the manipulator can reach. Many researchers published several works on the problem of computing these complete sets for robot kinematics. Based on the early studies [1, 2], several methods for workspace determination have been proposed, but many of them are applicable only to a particular class of robots. The workspace of parallel robots mainly depends 10 on the actuated joint variables, the range of motion of the joints and the mechanical interferences between the bodies of the mechanism. There are different techniques based on geometric [3, 4], discretization [5, 6, 7], and algebraic methods $[8,9,10,11,12]$ which can be used to compute the workspace of parallel robot. The main advantage of the geometric approach is that it establishes the 15 nature of the boundary of the workspace [13]. Also, it allows to compute the surface and volume of the workspace while being very efficient in terms of storage space, but when the rotational motion is included, it becomes less efficient. Interval analysis based methods can be used to compute the workspace but the computation time depends on the complexity of the robot and the requested 20 accuracy [7]. Discretization methods are usually less complicated and can easily take into account all kinematic constraints, but they require more space and computation time for higher resolutions. The majority of numerical methods used to determine the workspace of parallel manipulators includes the discretization of the pose parameters for computing workspace boundaries [6]. There 25 are other approaches, such that optimization algorithms [14] for fully serial or parallel manipulators; analytic methods for symmetrical spherical mechanisms [15]. In [16], a method for computing the workspace boundary for manipulators with a general structure is proposed, which uses a branch-and-prune technique to isolate a set of output singularities, and then classifies the points on such set according to whether they correspond to motion impediments in the workspace. A Cylindrical Algebraic Decomposition (CAD) based method is used in $[10,17,18]$ to model the workspace and joint space for the 3-R $\underline{P S}$ parallel robot and delta-like robots. The variations in the workspace, singularities, and joint space with respect to design parameter of a 3 -RPS parallel manipulator is 35 studied in [19]. 
Here, this paper presents the results obtained by applying algebraic methods for the workspace and joint space analysis of a family of a delta-like robot including complexity information for representing the singularities in the workspace and the joint space. The CAD algorithm is used to study both the workspace 40 and joint space, and a Gröbner based elimination process is used to compute the parallel and serial singularities of the manipulator. The structure of the paper is as follows. Section 2 presents the mathematical tools and the introduction of SIROPA. Section 3 describes the architecture of the manipulator, including kinematic equation and joint constraints associated with the manipulators. Sec-

45 tion 4 discusses the computation of parallel as well as serial singularities and their projections in workspace and joint-space. Section 5 and 6 present a comparative study on the shape of the workspace and joint space of different delta-like robots, respectively. Section 7 finally concludes the paper.

\section{Algebraic Tools : SIROPA}

SIROPA is a library for the MAPLE developed to analyze the singularities, workspace and joint space of serial and parallel manipulators as well as tensegrity structures [20]. There are two main parts of the library shown in Fig.(1), the first one provides the algebraic tools to solve the constraint equations and convert the trigonometric equations in the algebraic form. The other one, SIROPA, provides modeling, analyzing and plotting functions for different manipulators, shown in Fig.(2). Only a small part of these tools are used in the current paper.

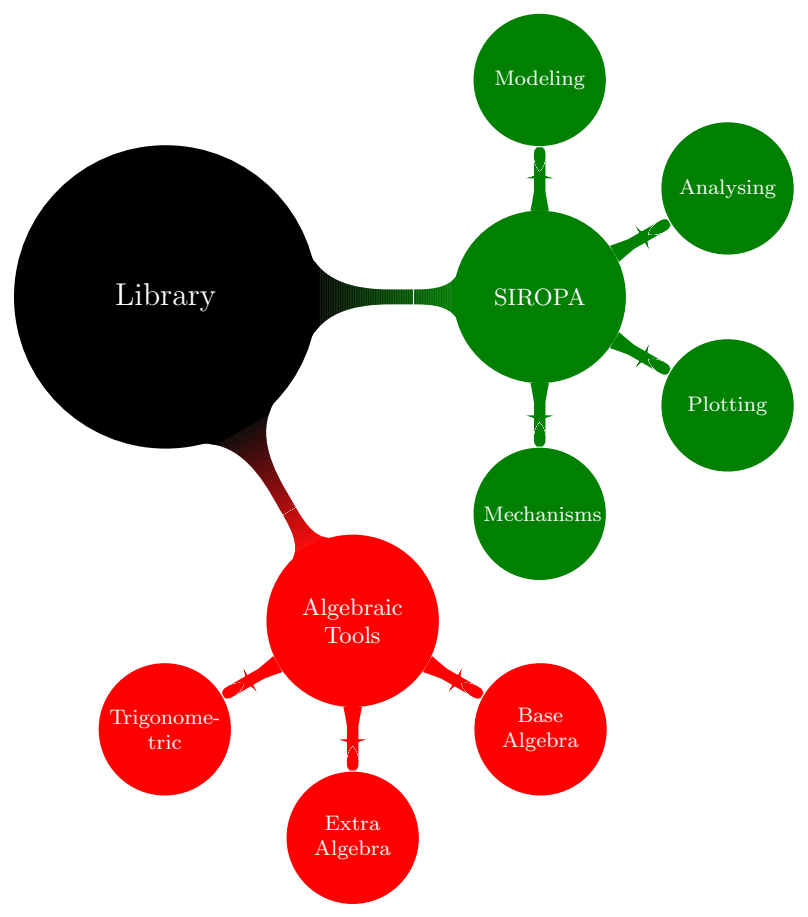

FiguRE 1: Architecture of Library 


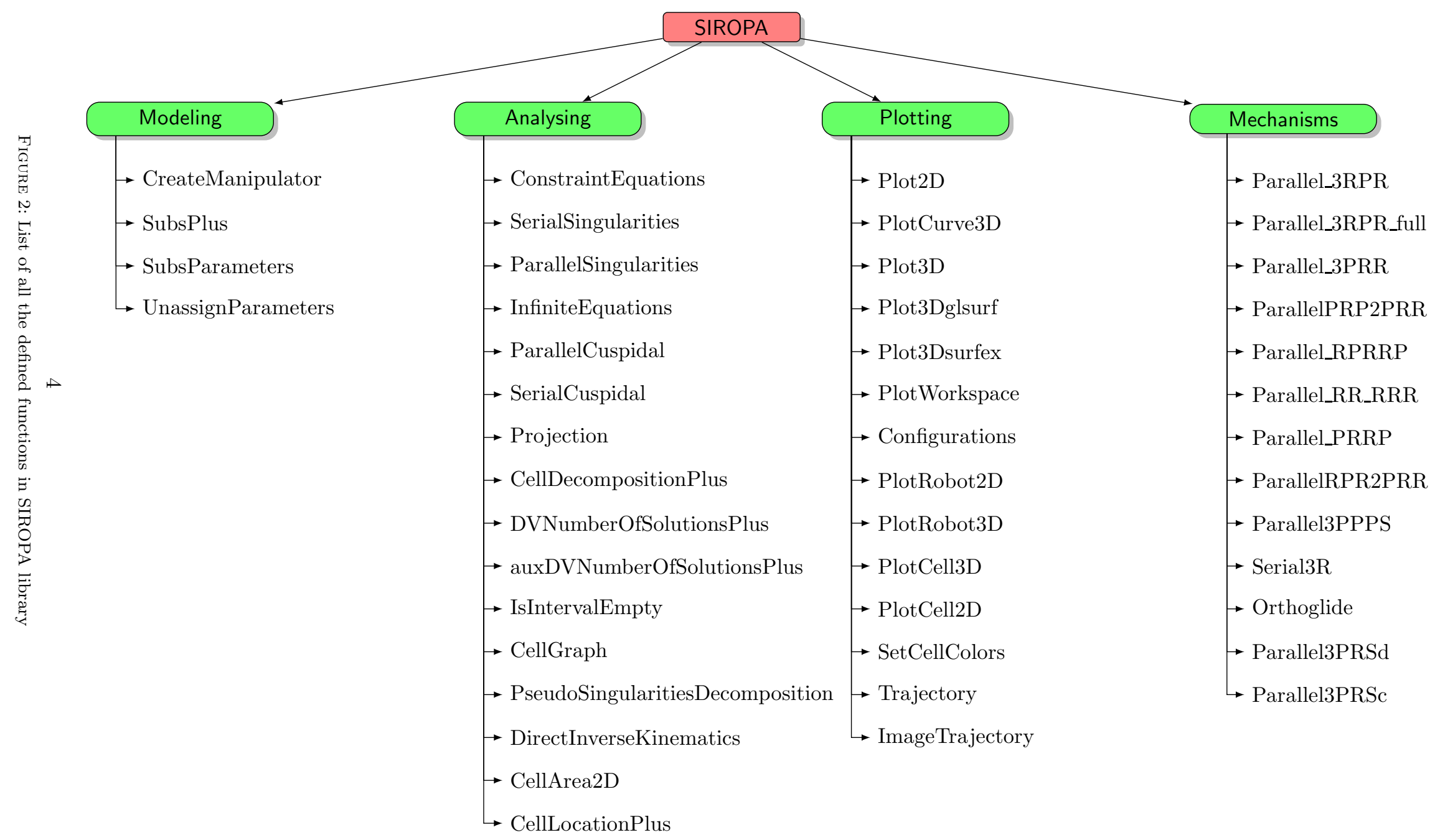




\subsection{Modeling Functions}

SIROPA provides modeling functions such as CreateManipulator(), to virtually create the planar and spatial manipulators for further analysis. Below are the functions :

\section{Functions}

CreateManipulator

SubsPlus

UnassignParameters

UnassignParameters
Constructs a data structure of type Manipulator Substitute coherently angles in a system

Specify parameter values in a Manipulator Release parameters in a Manipulator

\section{CreateManipulator}

The function CreateManipulator() of SIROPA library in MAPLE software is used to virtually create the manipulator for analysis. Listing 1 shows the code architecture of the function.

65

As shown in Listing 1. compulsory inputs are identified as [c], while the optional ones as [o]. Points, loops, chains and actuators, are the input parameters to create the plot of the manipulator. The pose variables are the essential input parameters to define the mechanism. The input parameter sys is the set

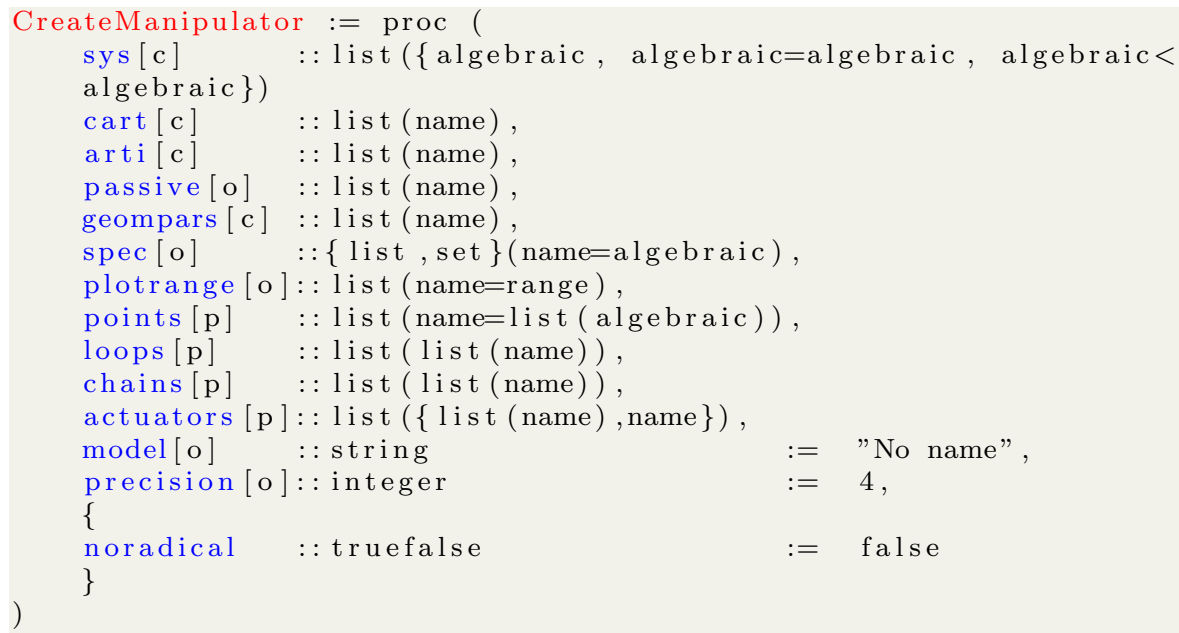

Listing 1: Architecture of Create Manipulator

This function returns a data structure of type Manipulator containing the fields, briefly described below. Further, the values of these fields can be retrieved or changed according to the analysis to be performed. 


\section{Parameters}

$\begin{array}{ll}\text { sys } & \text { list of polynomials, polynomial equalities and polynomial } \\ & \text { inequalities with rational coefficients : the implicit equa- } \\ & \text { tions and constraints of the considered manipulator } \\ & \text { list of names : the pose variables } \\ \text { cart } & \text { list of names : the control parameters ; default value : the } \\ \text { arti } & \text { names of sys not in vars } \\ \text { passive } & \text { list of names : the passive variables; default value : [] } \\ \text { geompars } & \text { list of names : the geometric parameters; default value : } \\ & \text { the names of sys not in cart, nor in arti } \\ \text { spec } & \text { a list of equations of the form name=formula where name } \\ & \text { is a parameter name and formula a polynomial with tri- } \\ & \text { gonometric function; the new variables in a formula are } \\ & \text { handled in the same way as the replaced variable. default } \\ & \text { value : [] } \\ \text { list of name=list : the points of the robot with their co- } \\ \text { ordinates; default value : [] } \\ \text { list of list of names : the frame loops of the robot default } \\ \text { value : [] } \\ \text { list of list of names : the frame chains of the robot default } \\ \text { value : [] } \\ \text { loops }\end{array}$

\section{Remarks}

${ }_{95}$ - Polynomials p appearing in sys, Equations, GenericEquations are considered implicitly as $\mathrm{p}=0$.

- When a control parameter value is specified in spec, the parameter name is removed from the ControlParemeters field. This is not the case for the geometric parameters that appears in the field GeometricParameters even if they are specified.

\subsection{Analysing Functions}

SIROPA provides the analysing function to compute the parallel and serial singularities. These functions are used to study both the workspace and joint space. The architecture of ConstraintEquations and CellDecompositionPlus are shown in Listing 2 and 3 .

\section{ConstraintEquations}

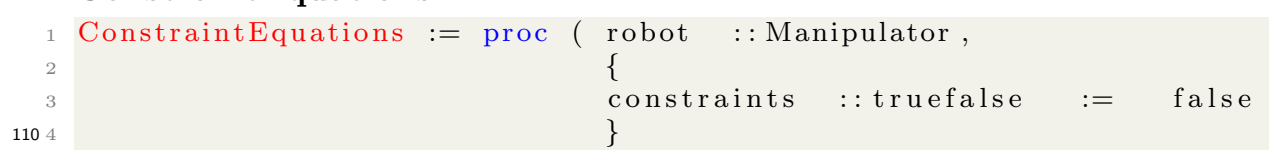




\section{Returns}

Equations

Constraints

ArticularVariables

PassiveVariables

GeometricParameters

GenericEquations

GenericConstraints

Precision

PoseValues

ArticularValues

PassiveValues

GeometricValues

DefaultPlotRanges

Points

Loops

Chains

Actuators

Model a list of polynomials $\left[p_{1}, \ldots, p_{k}\right]$ : the modeling equations

a list of strict inequalities : the constraint inequalities

a list of names : the control parameters

a list of names : the remaining variables

a list of names : the geometric parameters

a list of polynomials : the modeling equations with symbolic geometric parameters

a list of strict inequalities : the constraint inequalities appearing in sys

an integer : the number of correct digits

the pose values substituted in the GenericEquations to get the Equations

the articular values substituted in the GenericEquations to get the Equations

the passive values substituted in the GenericEquations to get the Equations

the geometric values substituted in the GenericEquations to get the Equations ranges used by default for plotting if provided the points coordinate of the robot the frame loops of the robot the frame chain of the robot the actuators of the robot a string : the name of the modeling

Listing 2: Architecture of ConstraintEquations function

Computes the implicit equations induced by the constraints.

Parameters

robot a data structure returned by a function mechanisms.

\section{Returns}

A list of list of polynomials : each list represents a component of the equations satisfied by the constraints.

\section{CellDecompositionPlus}

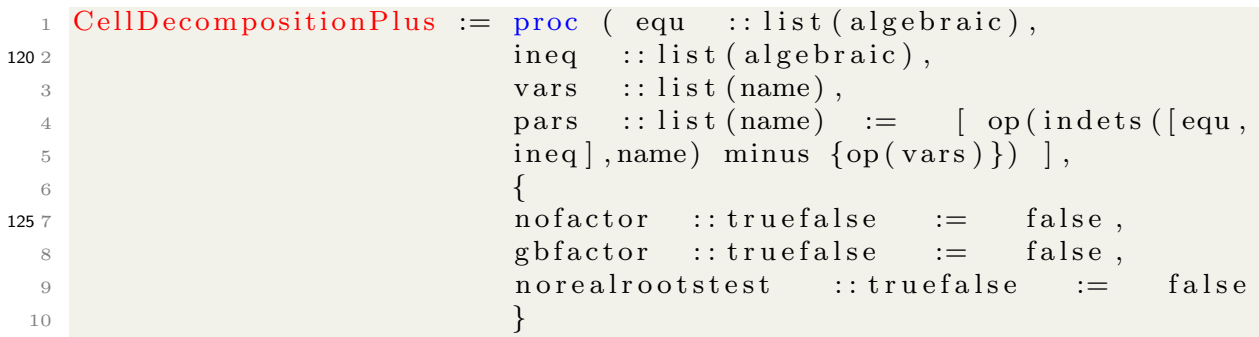




\section{Functions}

ConstraintEquations

SerialSingularities

ParallelSingularities

InfiniteEquations

ParallelCuspidal

SerialCuspidal

Projection

CellDecompositionPlus

NumberOfSolutionsPlus

DVNumberOfSolutionsPlus

auxDVNumberOfSolutionsPlus

IsIntervalEmpty

CellGraph

DirectInverseKinematics

CellArea2D

CellLocationPlus
Computes the implicit equations induced by the constraints.

Computes the implicit equations satisfied by the singularities.

Computes the implicit equations satisfied by the singularities.

Computes the equations where the manipulator has infinitely many solutions. Computes the implicit equations satisfied by the cuspidal points.

Computes the implicit equations satisfied by the cuspidal points.

Project on variables or expressions in a polynomial system.

Describes the parameter space according to the number of real roots.

Returns the number of real solutions of cells obtained by CellDecompositionPlus.

Returns the number of real solutions on the intersection points of the Discriminant Variety.

Auxiliary function Returns the number of real solutions on the intersection points of the Discriminant Variety.

Checks that a polynomial has no real roots in an open interval.

Computes the connexity graph of the cells of a CAD.

Returns the cells having the same antecedent.

Compute the area of the cells returned CellDecomposition or CellDecompositionPlus.

Computes the cells of a decomposition containing the given points.

Listing 3: Architecture of CellDecompositionPlus function

Describes the parameter space according to the number of real roots.

\section{Returns}

A maple object : the same as the one returned by the maple function RootFinding[Parametric][CellDecomposition]. The main difference is that it handles trigonometric expressions. 


\section{Parameters}

equ list of polynomials and trigonometric expressions : the
equations.
a list of polynomials and trigonometric expressions : the
inequalities where each expression $\mathrm{p}$ stands for $\mathrm{p}>0$.
a list of names : the variables of the system
a list of names : the parameters of the system; default
vars
value : the remaining variables of equ and ineq

\subsection{Plotting Functions}

These functions are used to plot the workspace, joint space and singularity surfaces. Listing 4, 5, 6] and 7] shows the architecture of Plot2D, Plot3D, Configurations and PlotRobot3D, respectively.

\section{Functions}

Plot2D

PlotCurve3D

Plot3D

Plot3Dglsurf

Plot3Dsurfex

PlotWorkspace

Configurations

PlotRobot2D

PlotRobot3D

PlotCell3D

PlotCell2D

SetCellColors

Trajectory

ImageTrajectory
Plots a system of 2 variables

Plots a curve given by implicit equations in 3 variables Plots a system of 3 variables using maple internal plotting functions

Plots a system of 3 variables using glsurf

Plots a system of 3 variables using surfex (software based on surf)

Plot the border of a manipulator workspace

Computes the different possible positions

Plot a planar manipulator

Plot a 3D manipulator

Plot the cells returned CellDecomposition or CellDecompositionPlus

Plot the cells returned CellDecomposition or CellDecompositionPlus

Set colors to the numbers of solutions obtained by NumberOfSolutionsPlus

Display a given trajectory

Display a given trajectory

\section{Plot2D}

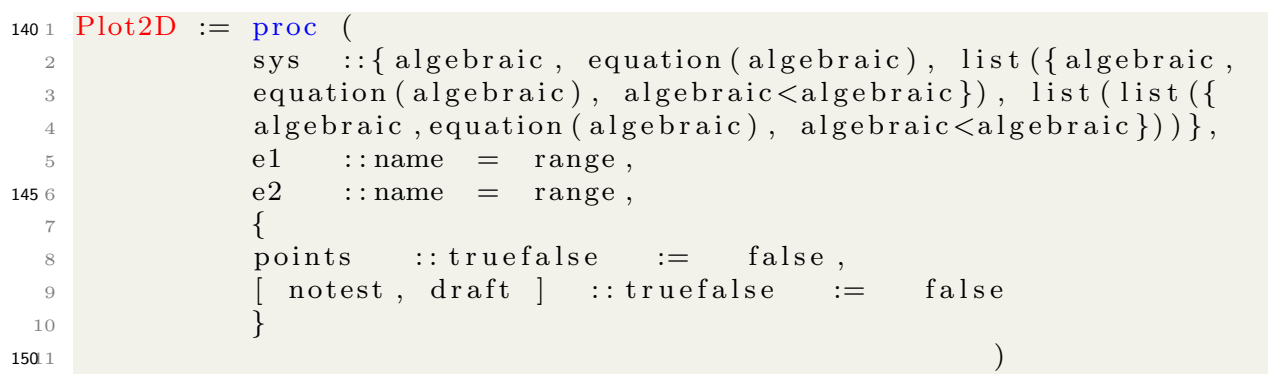

Listing 4: Architecture of Plot2D function 
Plots a system of 2 variables.

\section{Parameters}

$\begin{array}{ll}\text { sys } & \text { a list or a list of list of polynomials : the system } \\ \mathrm{v} 1=\mathrm{r} 1 & \mathrm{v} 1 \text { is a name of sys and } \mathrm{r} 1 \text { a range of values } \\ \mathrm{v} 2=\mathrm{r} 2 & \mathrm{v} 2 \text { is a name of sys and } \mathrm{r} 2 \text { a range of values } \\ \text { points }=\text { bool } & \text { bool is a boolean : if false, isolated points are ignored; } \\ \text { default value : false ; } \\ \text { notest }=\mathrm{b} & \mathrm{b} \text { is a boolean; when } \mathrm{b} \text { is true the inequality and real } \\ & \text { constraints are ignored; default value : true; } \\ \text { opts } & \text { arguments passed to the maple function plots :- } \\ & \text { implicitplot }\end{array}$

\section{Returns}

A graphic : the solutions of the system,

- when sys is a list of polynomials $[\mathrm{p} 1, \ldots, \mathrm{pk}]$, the graphic is the zeroes of the system $\mathrm{p} 1=0$ and $\ldots$ and $\mathrm{pk}=0$

- When sys is a list of list of polynomials $[\mathrm{L} 1, \ldots, \mathrm{Lk}]$, the graphic is the union of the zeroes of each system L1, ..., Lk.

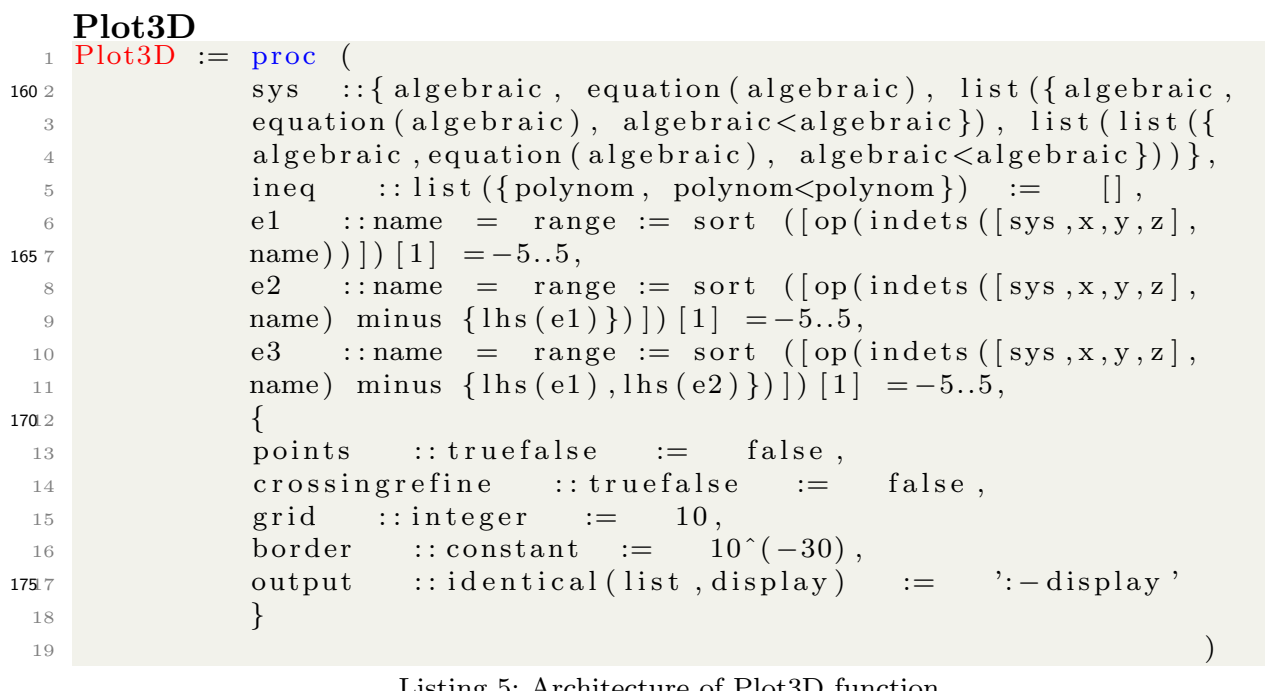

Plots a system of 3 variables using maple internal plotting functions.

\section{Returns}

A graphic : the solutions of the system,

- when sys is a polynomial, the graphic is the zeroes of this polynomial

— when sys is a list of polynomials $[\mathrm{p} 1, \ldots, \mathrm{pk}]$, the graphic is the zeroes of the system $\mathrm{p} 1=0$ and $\ldots$ and $\mathrm{pk}=0$

- when sys is a list of list of polynomials $[\mathrm{L} 1, \ldots, \mathrm{Lk}]$, the graphic is the union of the zeroes of each system L1, ..., Lk. 


\section{Parameters}

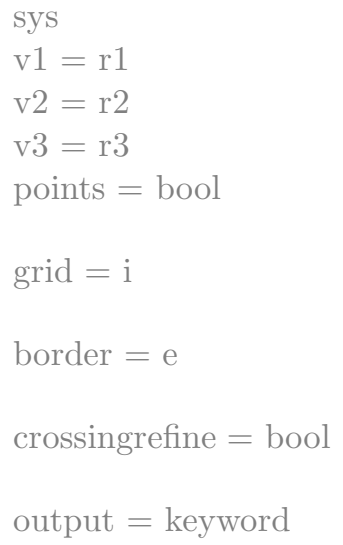

a list or a list of list of polynomials : the system $\mathrm{v} 1$ is a name of sys and $\mathrm{r} 1$ a range of values $\mathrm{v} 2$ is a name of sys and $\mathrm{r} 2$ a range of values $\mathrm{v} 3$ is a name of sys and $\mathrm{r} 3$ a range of values bool is a boolean : if false, isolated points are ignored; default value : false;

$\mathrm{i}$ is an integer leading to a grid size $\mathrm{i} x \mathrm{i}$; default value : 20 .

e is a numeric value : defines the precision on the border; default value : 0.0001 .

bool is a boolean : if true, the mesh follows the cross of the different surfaces; default value : false. keyword is either list or display : display (resp. list) returns a graph (resp. a list).

\section{Configurations}

This function computes the different possible working modes for given values of pose variables and assembly modes for given values of articular variables.

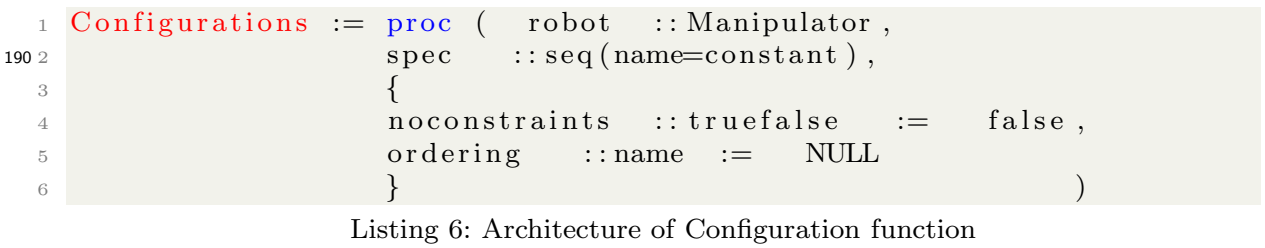

Computes the different possible positions.

\section{Parameters}

$\begin{array}{ll}\text { robot } & \text { an object of type Manipulator } \\ \text { spec } & \text { sequence of name=constant }: \text { the specification of the } \\ & \text { known variables (the articular values or the pose } \\ & \text { values, or other) } \\ \text { noconstraints }={ }^{*} \mathrm{~b}^{*} & \mathrm{~b} \text { is a boolean : when true, the constraint inequali- } \\ & \text { ties are ignored; default value : false. } \\ \text { ordering } & \text { ordering is a name used to order the solutions ; de- } \\ & \text { fault value : NULL }\end{array}$

\section{Returns}

A list of elements : each elements is a list of name=list(constant) and represents a configuration of the input manipulator. 


\section{PlotRobot3D}

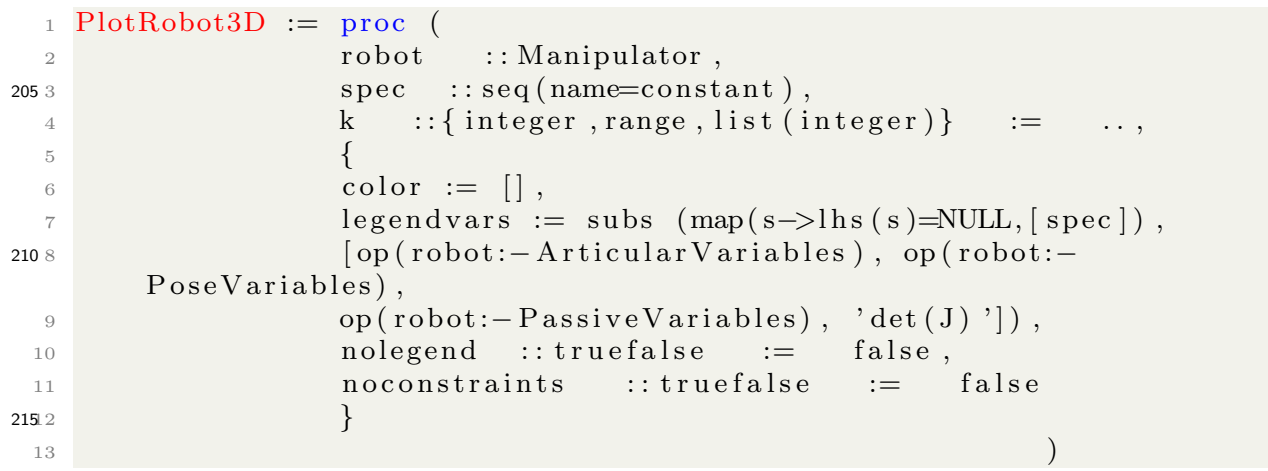

Listing 7: Architecture of PlotRobot3D function

Computes the different possible positions.

\section{Parameters}

\begin{tabular}{|c|c|}
\hline robot & a Manipulator : the $3 \mathrm{D}$ robot to plot \\
\hline spec & $\begin{array}{l}\text { a sequence of name }=\text { constant }: \text { specification of variables } \\
\text { of the robot to plot }\end{array}$ \\
\hline $\mathrm{k}$ & $\begin{array}{l}\text { an integer : specifies one of the possible configuration } \\
\text { when several are available }\end{array}$ \\
\hline color $=$ col & $\begin{array}{l}\text { equation of the shape color }=^{*} \mathrm{col}^{*} \text {, where col is a color or } \\
\text { a list of colors; when the number of specified color is not } \\
\text { enough, deterministic colors are chosen; default value : } \\
\text { empty list. }\end{array}$ \\
\hline legendvars & $\begin{array}{l}\text { list of names : the variables to display in the legend; de- } \\
\text { fault value : the articular, passive and pose variables, mi- } \\
\text { nus the variables in spec }\end{array}$ \\
\hline nolegend $=\mathrm{b}$ & $\begin{array}{l}\mathrm{b} \text { is a boolean : when false, a legend is displayed (the gra- } \\
\text { phic appears in a separate windows with a classic work- } \\
\text { sheet); default value : false. }\end{array}$ \\
\hline
\end{tabular}

\section{Returns}

A graphic : the different configurations of the manipulator satisfying the input specifications.

\subsection{Mechanisms Functions}

There are some manipulators like 3-RPR (see Listing 8)., 3-PRR, R $\underline{P R R} \underline{P}, 3-$

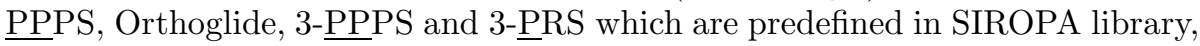
and can be accessible using these functions [21, 22, 23, 24]. 


\section{Functions}

Parallel_3RPR

Parallel_3RPR_full

Parallel_3PRR

ParallelPRP2PRR

Parallel_RPRRP

Parallel_RR_RRR

Parallel_PRRP

Orthoglide

ParallelRPR2PRR

Parallel3PPPS

Serial3R

Parallel3PRSd

Parallel3PRSc
Constructs the Manipulator object of planar 3-RPR Constructs the Manipulator object of planar 3-R $\underline{P R}$ Constructs the Manipulator object of a $3-\underline{P R R}$ Constructs the Manipulator object of a PRP2PRR Constructs the Manipulator object of a RPRR$\underline{P}$ Constructs the Manipulator object of a 2-RR Constructs the Manipulator object of a $\underline{P R R} \underline{P}$ Constructs the Manipulator object of Orthoglide Constructs the Manipulator object of the RPR2PRR

Constructs the Manipulator object of the 3-PPPS Constructs the Manipulator object of the serial 3R manipulator.

Constructs the Manipulator object of the 3 - $\underline{P R S}$

Constructs the Manipulator object of the 3-PRS

225 Parallel_3RPR

Parallel_3RPR := proc(

d1 : : algebraic $:=\quad 17.04$

d2 : : algebraic $:=16.54$

d3 : : algebraic $:=20.84$,

beta : algebraic $:=\arccos \left(\left(\mathrm{d} 2 \wedge 2-\mathrm{d} 33^{\wedge} 2-\right.\right.$

$\left.\left.\mathrm{d} 1 \wedge^{\wedge} 2\right) /(-2 * \mathrm{~d} 3 * \mathrm{~d} 1)\right)$,

$\mathrm{A} 2 \mathrm{x} \quad:$ : algebraic $:=15.91$,

A3x $\quad:$ algebraic $:=0$,

A3y ::algebraic $:=10$,

precision : : integer $:=4$

\}

morespec : : seq (name=algebraic),

moreranges $\quad:$ seq (name $=$ range $)$

Listing 8: Architecture of Parallel_3RPR function

Constructs the Manipulator object of a planar 3-RPR manipulator.

\section{Parameters}

name $=$ constant

precision $=$ integer the geometric parameters of the robot (see Fig.(13)),

- where name is one of $\mathrm{d} 1, \mathrm{~d} 2, \mathrm{~d} 3$, beta, $\mathrm{A} 2 \mathrm{x}$, A3x, A3y,

- All the variables d1, d3, A2x, A3x, A3y must be assigned.

- One of d2, beta must be assigned (if both are assigned, d3 is ignored).

- By default, the values are $\mathrm{d} 1=17.04, \mathrm{~d} 2=$ $16.54, \mathrm{~d} 3=20.84, \mathrm{~A} 2 \mathrm{x}=15.91, \mathrm{~A} 3 \mathrm{x}=0$, $\mathrm{A} 3 \mathrm{y}=10$.

the precision, where integer is the number of significative digits; default value : 4 . 


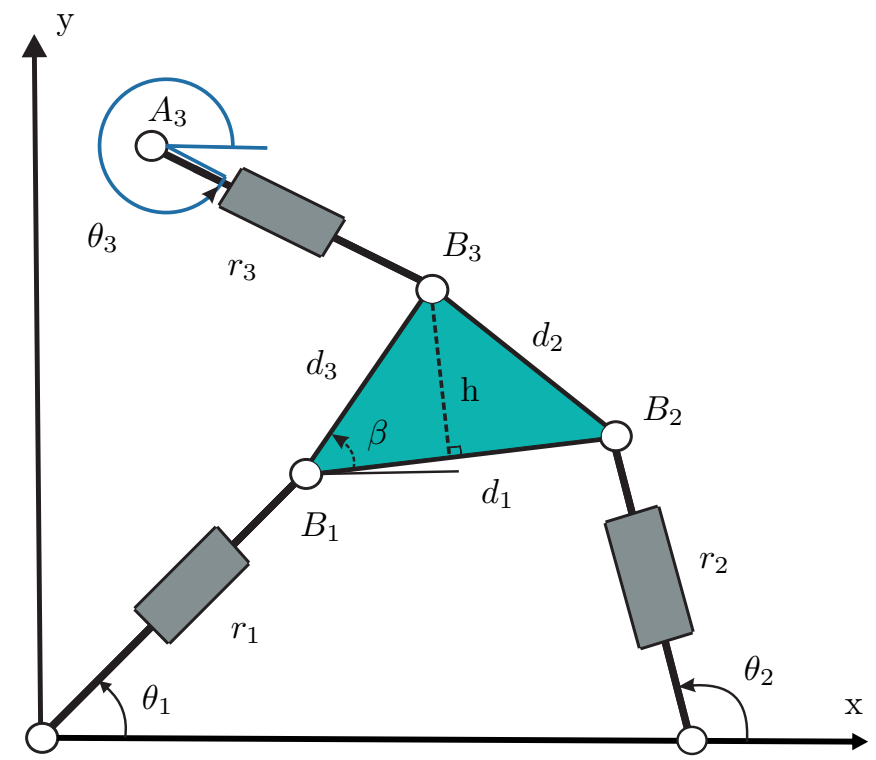

$A_{1}$

$A_{2}$

FiguRE 3: 3-R $\underline{P R}$ parallel robot

\section{Returns}

A Manipulator data structure representing the planar 3-RPR manipulator whose dimensions are given in input.

\subsection{Standard Bases (Gröbner Bases)}

The method of Gröbner bases provides a uniform approach to solve a wide range of problems expressed in terms of sets of multivariate polynomials. The Gröbner basis gives us a method for writing a system of algebraic equations $f\left(x_{1}, \ldots, x_{n}\right)=0$ in terms of unknowns $x_{1}, \ldots, x_{n}$ with finitely many solutions into a system that has the same roots and in a triangular form $g_{n}\left(x_{n}\right)=$ $0, g_{n-1}\left(x_{n-1}, x_{n}\right)=0, \ldots, g_{1}\left(x_{1}, \ldots, x_{n}\right)=0$, called a Gröbner basis. There are few drawbacks of Gröbner basis such that the calculation time of the Gröbner basis is mainly dependent upon the number of equations and their degree; while its calculation with real numbers is numerically unstable 25].

Gröbner basis theory can be used to compute the projections $\pi_{Q}$ and $\pi_{W}$ into the joint space and the workspace, respectively. Let $P$ be a set of polynomials in the variables $X=\left(x_{1}, . ., x_{n}\right)$ and $q=\left(q_{1}, . ., q_{n}\right)$. Moreover, let $V$ be the set of common roots of the polynomial in $P$, let $W$ be the projection of $\mathrm{V}$ on the workspace and $\mathrm{Q}$ the projection on the joint space. It might not be possible to represent $W$ (resp. $Q$ ) by polynomial equations. Let $\bar{W}(\operatorname{resp} . \bar{Q})$ be the smallest set defined by polynomial equations that contain $W$ (resp. $Q$ ) 11. A Gröbner basis $P$ is a polynomial system equivalent to $\mathrm{P}$, satisfying some additional specific properties. The Gröbner basis of a system depends on the chosen ordering of monomials.

For the projection $\pi_{Q}$, when we choose an ordering eliminating $\mathbf{q}$, the Gröbner basis of $\mathrm{P}$ contains exactly the polynomials defining $\bar{W}$. 


\begin{tabular}{|l|l|}
\hline sys & $\begin{array}{l}\text { list of equations and strict inequalities between } \\
\text { polynomials with rational coefficients }\end{array}$ \\
\hline vars & list of names; the indeterminates \\
\hline pars & (optional) list of names; the parameters \\
\hline eqs & $\begin{array}{l}\text { list of polynomials } f \text { with rational coefficients, } \\
\text { representing equations of the form } f=0\end{array}$ \\
\hline ineqs & $\begin{array}{l}\text { list of polynomials } g \text { with rational coefficients, } \\
\text { representing constraint inequalities of the form } 0<g\end{array}$ \\
\hline \hline
\end{tabular}

TABLE 1: Description of the fields of DiscriminantVariety function

For the projection $\pi_{W}$, when we choose an ordering eliminating $\mathbf{X}$, the Gröbner basis of $\mathrm{P}$ contains exactly the polynomials defining $\bar{Q}$.

\subsection{Discriminant Variety and Cylindrical Algebraic Decomposition}

The notion of discriminant variety is a generalization of the discriminant of a univariate polynomial, describing all the critical points of a system, including singularities, solutions of multiplicity greater than one, and solutions at infinity. It is a subset of the parameter space of lower dimension [26, 27].

As shown in Table 1, a discriminant variety has the following property : it divides the parameter space into open, full-dimensional cells such that the number of solutions of the system sys is constant for parameter values chosen from the same open cell. As shown in Listing 9, the function DiscriminantVariety(sys, vars, pars) computes a discriminant variety of the system sys of equations and inequalities with respect to the indeterminates vars and the parameters pars.

2801 DiscriminantVariety (sys, vars, pars)

2 DiscriminantVariety (eqs, ineqs, vars, pars)

Listing 9: Architecture of Discriminant Variety

The function DiscriminantVariety(eqs, ineqs, vars, pars) computes a discriminant variety of the system

$$
[f=0,0<g]_{f \in e q s,} \quad g \in \text { ineqs }
$$

of equations and inequalities with respect to the indeterminates vars and the parameters pars.

The input system must satisfy the following properties :

- There are at least as many equations as indeterminates.

- At least one and at most finitely many complex solutions exist for almost all complex parameter values (the system is generically solvable and generically zero-dimensional).

- For almost all complex parameter values, there are no solutions of multiplicity greater than one (the system is generically radical). In particular, the input equations are square-free.

An error occurs if one of these three previous conditions is violated. 
- The result is returned as a list of lists of polynomials in pars such that the discriminant variety is the union of the set of solutions of the polynomials in each inner list.

- If pars is not specified, it defaults to all the names in sys that are not indeterminates.

- This function attempt to find a minimal discriminant variety, but it may return a proper superset in the case that it does not succeed.

- The discriminant variety is computed using Gröbner basis techniques.

\section{Example 1}

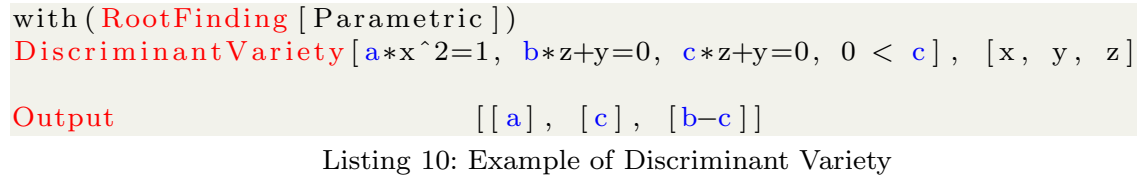

The discriminant variety in Listing 10 is $(a, b, c): a=0$ or $b=c$ or $c=0$. The case $a=0$ gives a solution of the first equation at infinity. In the case $b=c$, the second and third equations coincide and therefore the system becomes underdetermined and has infinitely many solutions. Finally, the case $c=0$ corresponds to a boundary case for the inequality $0<c$.

A cylindrical algebraic decomposition of the $\mathrm{n}$-dimensional real space is a partition of the whole space into connected semi-algebraic subsets such that the cells in the partition are cylindrically arranged, that is, the projection of any two cells onto any lower dimensional real space is either equal or disjoint. This decomposition is called F-invariant if, for any given cell, the sign of each polynomial in $\mathrm{F}$ does not change over the cell. CylindricalAlgebraicDecompose( $\mathrm{F}$, $\mathrm{R}$ ) returns an F-invariant $\mathrm{CAD}$ of the $n$-dimensional real space, where $n$ is the number of variables in $\mathrm{R}$. This assumes that $\mathrm{R}$ has characteristic zero and no parameters, such that the base field of $\mathrm{R}$ is the field of rational numbers [28].

The output of CylindricalAlgebraicDecompose $(F, R)$ has several possible formats controlled by the options output $=$ piecewise, tree, list , cadcell, rootof. In all formats, each cell provides at least two pieces of information; the index of the cell; and a sample point of the cell. In the cadcell and rootof output formats, a defining semi-algebraic system (called a Tarski Formula) is also provided. Due to the cylindicity property, cells can be organized in a hierarchical manner. This is the purpose of piecewise and tree output format, whereas the other three formats are flat representations. Due to the potentially large number of cells, the cadcell format only shows the name cadcell for each cell in the decomposition. However, cadcell is a type and an object of that type can be passed to Display. It can also be passed to SamplePoints in order to access the sample point of the cell. The rootof format is meant to be compatible with the output format of the solve command.

As shown in Listing 11 and Table 2, the CellDecomposition function decomposes the parameter space of a parametric polynomial system into cells in which the original system has a constant number of solutions [29]. 


\begin{tabular}{|l|l|}
\hline sys & $\begin{array}{l}\text { list of equations and strict inequalities between } \\
\text { polynomials with rational coefficients }\end{array}$ \\
\hline vars & list of names; the indeterminates \\
\hline pars & (optional) list of names; the parameters \\
\hline eqs & $\begin{array}{l}\text { list of polynomials } f \text { with rational coefficients, } \\
\text { representing equations of the form } f=0\end{array}$ \\
\hline posineqs & $\begin{array}{l}\text { list of polynomials } g \text { with rational coefficients, } \\
\text { representing constraint inequalities of the form } 0<g\end{array}$ \\
\hline nzineqs & $\begin{array}{l}\text { list of polynomials } g \text { with rational coefficients, } \\
\text { representing constraint inequations of the form } g \neq 0\end{array}$ \\
\hline options & $\begin{array}{l}\text { sequence of optional equations of the form keyword }=\text { value, } \\
\text { where keyword is either output or method }\end{array}$ \\
\hline \hline
\end{tabular}

TABLE 2: Description of the fields of CellDecomposition function

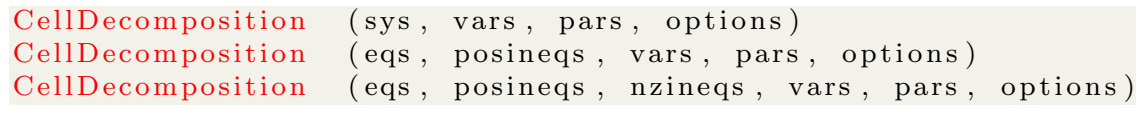

The function returns a data structure that can be used for (examples) :

- Plotting the regions of the parameter space for which the system has a given number of solutions.

- Extracting sample points in the parameter space for which the system has a given number of solutions.

— Extracting boxes in the parameter space in which the system has a given number of solutions.

The record returned captures information about the solutions of the system depending on the parameter values, including :

- a discriminant variety;

— for each full-dimensional open cell, a sample point strictly in the interior of the cell; if possible, the coordinates of the sample point are chosen to be integers.

The input system must satisfy the following properties :

- The number of equations is equal to or greater than the number of indeterminates ;

- At most finitely many complex solutions exist for almost all complex parameter values (the system is generically zero-dimensional);

- For almost all complex parameter values, there are no solutions of multiplicity greater than one (the system is generically radical); in particular, the input equations are square-free.

\section{Manipulators Under Study}

There are four different mechanisms for which the workspace, the joint space and the singularities are presented in this paper. Three degree of freedom parallel mechanisms consisting of three identical legs, while the different arrangements of these legs give rise to family of delta like robot. Several types of delta-like robot were studied, few of them are Orthoglide [7, 30], Hybridglide, Triaglide 
[31] and UraneSX [7]. The kinematic equations of the family of delta like robot can be generalized as $\left\|\mathbf{P}-\mathbf{B}_{i}\right\|=L_{i}$. These constraint equations can be in the form of Euler angle representation or quaternions [10]. All the computations and analysis are done for $L_{i}=L=2$ and by imposing the following constraints on joint variables. Without joint limits, the whole family of these robots admit two assembly modes and eight working modes, i.e. ,

$$
0<\rho_{1}<2 L \quad 0<\rho_{2}<2 L \quad 0<\rho_{3}<2 L
$$

\subsection{Orthoglide Architecture and Kinematics}

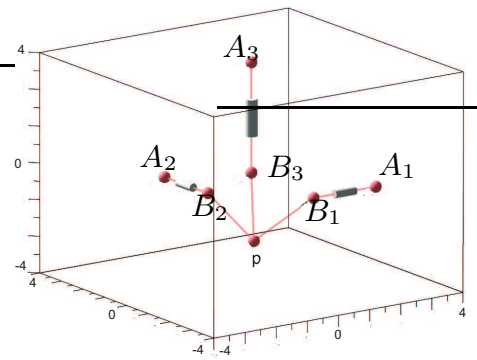

(a)

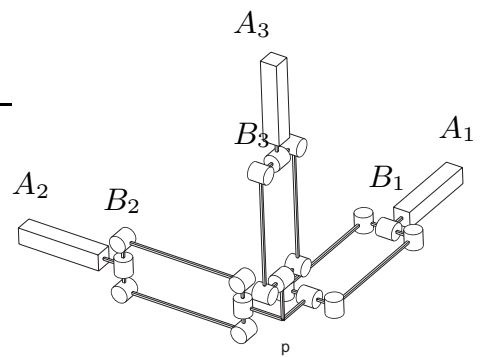

(b)

Figure 4: Configuration of Orthoglide : (a) simplified; (b) real.

As shown in Fig.(4), the Orthoglide mechanism is driven by three actuated orthogonal prismatic joints. A simpler model can be defined for the Orthoglide, namely, three bar links connected by the revolute joints to the tool center point on one side and to the corresponding prismatic joint at another side. Several assembly modes of these robots depends upon the solutions of direct kinematic problem (DKP). The point $\mathbf{P}$ represents the pose of corresponding robot. However, more than one position for the point $\mathbf{P}$ shows the multiple solutions for the DKP. $\mathbf{A}_{i} \mathbf{B}_{i}$ is equal to $\rho_{i}$, where $\rho_{i}$ represents the prismatic joint variables, whereas $\mathbf{P}$ represents the position vector of the tool center point. The constraint equations for the Orthoglide are :

$$
\begin{aligned}
& \left(x-\rho_{1}\right)^{2}+y^{2}+z^{2}=L^{2} \\
& x^{2}+\left(y-\rho_{2}\right)^{2}+z^{2}=L^{2} \\
& x^{2}+y^{2}+\left(z-\rho_{3}\right)^{2}=L^{2}
\end{aligned}
$$

The Maple lines used to describe this robot are as follows, shown in Listing 12

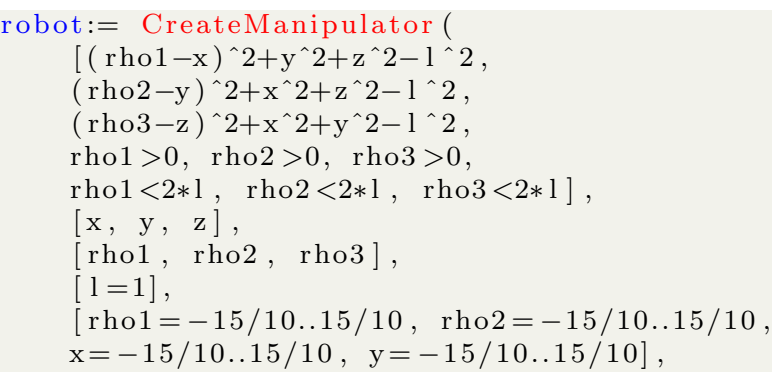




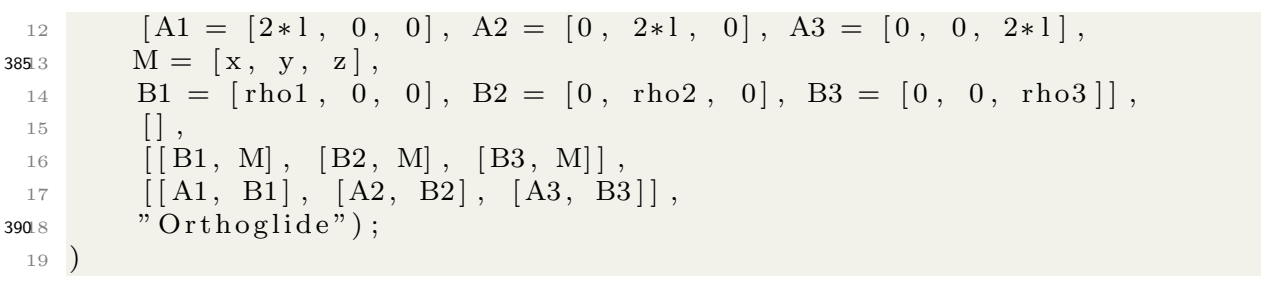

Listing 12: Maple lines to create the Orthoglide robot

\subsection{Hybridglide Architecture and Kinematics}

As shown in Fig. (5), the Hybridglide mechanism consists of three actuated prismatic joints, in which two actuators are placed parallel and third one perpendicular to others two. Also the three bar links connected by spherical joints to the tool center point on one side and to the corresponding prismatic joint at another side. Several assembly modes of these robots depends upon the solutions of the DKP.

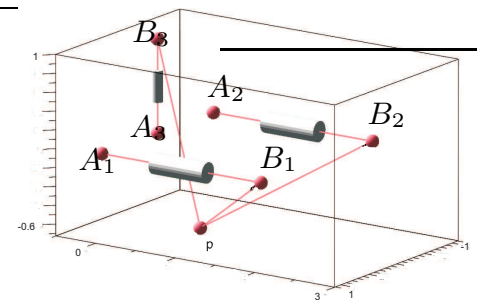

(a)

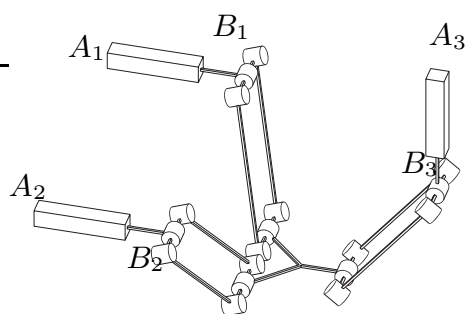

(b)

Figure 5: Configuration of Hybridglide : (a) simplified; (b) real.

The constraint equations for the Hybridglide are :

$$
\begin{aligned}
(x-1)^{2}+\left(y-\rho_{1}\right)^{2}+z^{2} & =L^{2} \\
(x+1)^{2}+\left(y-\rho_{2}\right)^{2}+z^{2} & =L^{2} \\
x^{2}+y^{2}+\left(z-\rho_{3}\right)^{2} & =L^{2}
\end{aligned}
$$

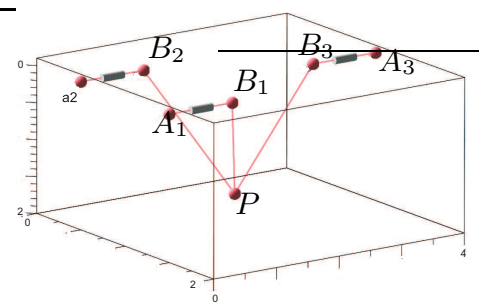

(a)

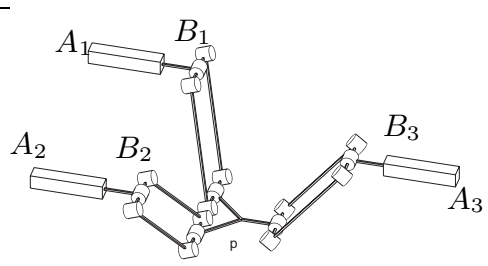

(b)

Figure 6: Configuration of Triaglide: (a) simplified; (b) real. 
As shown in Fig.(6), the Triaglide manipulator is driven by three actuated prismatic joints, in which all the three actuators all parallel to each other and placed in the same plane.

The constraint equations for the Triaglide are :

$$
\begin{aligned}
(x-1)^{2}+\left(y-\rho_{1}\right)^{2}+z^{2} & =L^{2} \\
(x+1)^{2}+\left(y-\rho_{2}\right)^{2}+z^{2} & =L^{2} \\
x^{2}+\left(y-\rho_{3}\right)^{2}+z^{2} & =L^{2}
\end{aligned}
$$

\subsection{UraneSX Architecture and Kinematics}

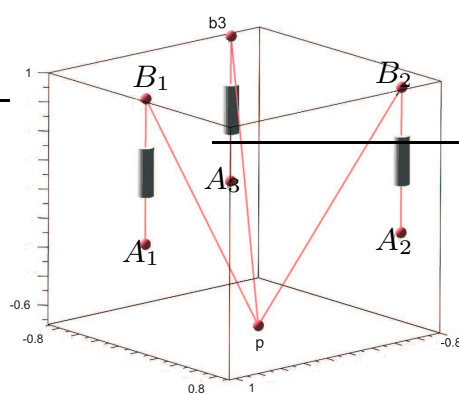

(a)

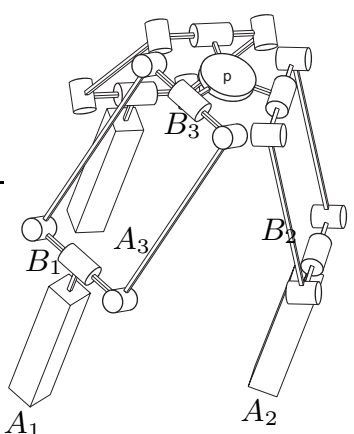

(b)

FIGURE 7: Configuration of UraneSX : (a) simplified; (b) real.

As shown in Fig.(7), the UraneSX is similar to triaglide, but instead of three actuators in the same plane, they are placed in different planes. The constraint equations for the UraneSX are :

$$
\begin{aligned}
(x-1)^{2}+y^{2}+\left(z-\rho_{1}\right)^{2} & =L^{2} \\
(x+1 / 2)^{2}+(y-\sqrt{3} / 2)^{2}+\left(z-\rho_{2}\right)^{2} & =L^{2} \\
(x+1 / 2)^{2}+(y+\sqrt{3} / 2)^{2}+\left(z-\rho_{3}\right)^{2} & =L^{2}
\end{aligned}
$$

\section{Singularities : Delta-Like Family Robot}

Singularities of a robotic manipulator are important feature that essentially influence its motion capabilities. Mathematically, a singular configuration may be defined as rank deficiency of the Jacobian describing the differential mapping from the joint space to the workspace and vice versa. Differentiating the constraints equations with respect to time leads to the following velocity relationship :

$$
\text { At }+\mathbf{B} \dot{\mathbf{q}}=0
$$

where $\mathbf{A}$ and $\mathbf{B}$ are the parallel and serial Jacobian matrices, respectively, $\mathbf{t}$ is the velocity of $\mathbf{P}$ and $\dot{\mathbf{q}}$ joint velocities. The parallel singularities occur whenever $\operatorname{det}(\mathbf{A})=0$, while the serial singularities occur whenever $\operatorname{det}(\mathbf{B})=0$. 


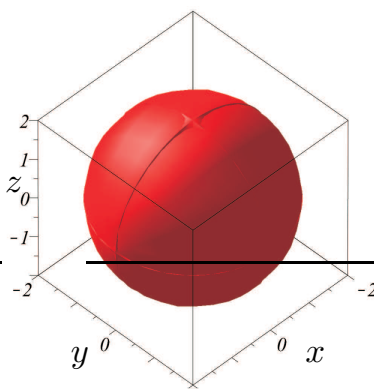

(a)

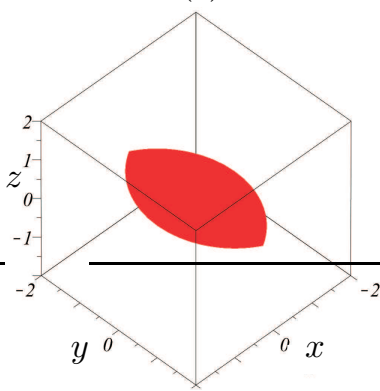

(c)

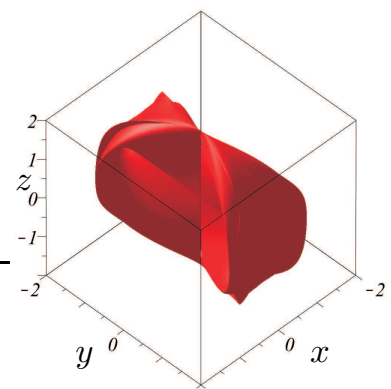

(b)

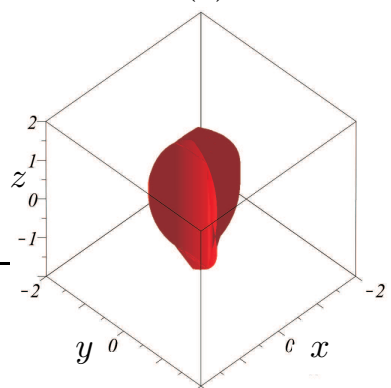

(d)

Figure 8: Projection of parallel singularity surface in the workspace for (a) Orthoglide, (b) Hybridglide, (c) Triaglide and (d) UraneSX

\subsection{Parallel Singularities : Projection in workspace and joint space}

Parallel singularities occur when the determinant of the direct kinematics matrix A vanishes. The corresponding singular configurations are located inside the workspace. They are particularly undesirable because the manipulator can not resist any force and control is lost. Parallel singularity and its projection surfaces in workspace and joint space are calculated using the function ParallelSingularities(). Listing 13 shows an example for calculating the singularity surfaces and its projection in joint space.

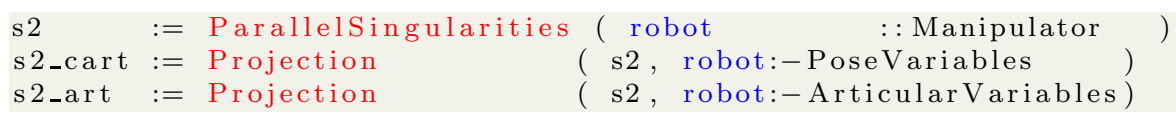

Listing 13: Projection of parallel singularity surface of delta-like robot in workspace and joint space

Parallel singularities and their projections in workspace and joint space are computed using a Gröbner based elimination method. This usual way for eliminating variables (see [32]) computes (the algebraic closure of) the projection of the parallel singularities in the workspace.

Eliminating variables can be done in favorable case by using cascading resultants. The main related result says that given two polynomials $P, Q \in$ ${ }_{430} \mathbb{C}\left[x_{1}, \ldots, x_{n}\right]$ and their resultant $R \in \mathbb{C}\left[X_{1}, \ldots, x_{n-1}\right]$ and $\alpha=\left(\alpha_{1}, \ldots, \alpha_{n-1}\right) \in$ $\mathbb{C}$ such that $R(\alpha)=0$, then, either $\alpha$ cancels the leading terms of $P, Q$ in $x_{n}$ 


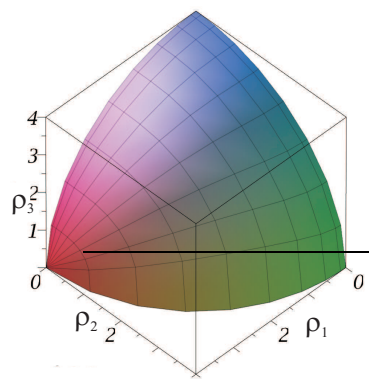

(a)

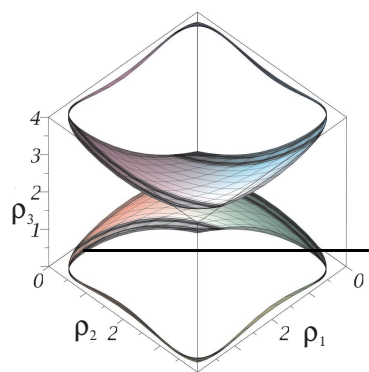

(c)

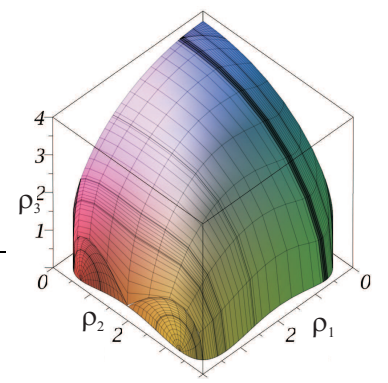

(b)

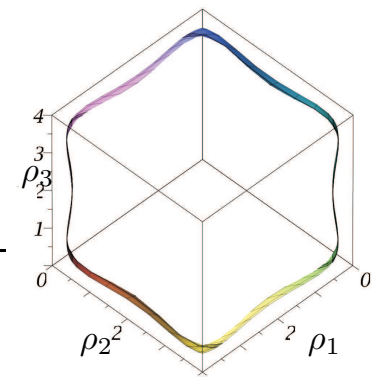

(d)

Figure 9: Projection of parallel singularity surfaces in the joint space for (a) Orthoglide, (b) Hybridglide, (c) Triaglide and (d) UraneSX

(which means that some parts of the varieties $P=0$ or $Q=0$ are $\ll$ going to infinity $\gg$ or $\exists \alpha_{n} \in \mathbb{C}$ such that $P\left(\alpha_{1}, \ldots, \alpha_{n}, \alpha_{n+1}\right)=Q\left(\alpha_{1}, \ldots, \alpha_{n}, \alpha_{n+1}\right)=0$. Some linear change of variables allows to ignore the parts « going to infinity $\gg$ and $\left\{x \in C^{n-1}, R(x)=0\right\}$ is then the projection of $V(P, Q)=\{x \in$ $\left.\mathbb{C}^{n}, P(x)=Q(x)=0\right\}$. One can generalize this elimination step to sets of more than two polynomials.

Taking pairs of resultants and computing their resultant with respect to $x_{n-1}$ one then project again, eliminating one more variable, defining so a cascading process that will finish with univariate polynomials. This process has almost the same complexity as the projection step in a CAD adapted to the input polynomials : at each step, the degree of the polynomials is doubling inducing an exponential growth (double exponential in the number of variables).

On the contrary, in favorable situations (which is our case) the discriminant variety is known to induce a lower growth of coefficients and degrees (single exponential in the number of variables [33]).

Let have a look to the intersection of 3 algebraic surfaces $p_{1}\left(x_{1}, x_{2}, x_{3}\right)=$ $p_{2}\left(x_{1}, x_{2}, x_{3}\right)=p_{3}\left(x_{1}, x_{2}, x_{3}\right)=0$ of $\mathbb{C}^{3}$ defining a finite set of points. Such a system can also be viewed as the intersection of 3 space curves $p_{1}=p_{2}=0$ ${ }_{450}, p_{1}=p_{3}=0$ and $p_{2}=p_{3}=0$. By computing the resultant of each pairs of polynomials wrt $x_{3}$ one gets the projections of the space curves onto the coordinates $x_{1}, x_{2}$.

A point is that the intersection of these projections might contain more points than the projection of the intersections of the space curves. It is easy to 455 see that two space curve that do not intersect might have projections with non 
empty intersections. The cascading process might follow with the plane curves, but (numerous) spurious points might have been introduced.

$$
\begin{aligned}
& \operatorname{det}(\mathbf{A})_{o}=-\rho_{1} \rho_{2} \rho_{3}+\rho_{1} \rho_{2} z+\rho_{1} \rho_{3} y+\rho_{2} \rho_{3} x \\
& \operatorname{det}(\mathbf{A})_{h}=-\rho_{1} \rho_{3} x+\rho_{2} \rho_{3} x-\rho_{1} \rho_{3}+\rho_{1} z-\rho_{2} \rho_{3}+\rho_{2} z+2 \rho_{3} y \\
& \operatorname{det}(\mathbf{A})_{u}=\sqrt{3}\left(3 z-\rho_{1}-\rho_{2}-\rho_{3}+\rho_{3} x+\rho_{2} x-2 \rho_{1} x\right)+3 \rho_{3} y-12 \rho_{2} y \\
& \operatorname{det}(\mathbf{A})_{t}=\rho_{1} z+\rho_{2} z-2 \rho_{3} z
\end{aligned}
$$

In the same way, one can compute (the algebraic closure of) the projection of the parallel singularities in the joint space. Both are then defined as the zero set of some system of algebraic equations and we assume that the considered robots are generic enough so that both are hypersurfaces. $\operatorname{det}(\mathbf{A})_{o}, \operatorname{det}(\mathbf{A})_{h}, \operatorname{det}(\mathbf{A})_{t}$ and $\operatorname{det}(\mathbf{A})_{u}$ are the parallel singularities of Orthoglide, Hybridglide, Triaglide and UraneSX, respectively, as shown in Eq. (8). Starting from the constraint equations and the determinant of the Jacobian matrix, we are able to eliminate the joint values. This elimination strategy is more efficient than a cascading elimination by means of resultants which might introduce many more spurious solutions : singular points that are not projections of singular points.

Figure (9) shows the projections of singularity surface $s 2$ in jointspace and $s 2 \_a r t$ is the projection surface. And $s 2 \_a r t$ is the projection curve in workspace shown in Fig.(8).

\subsection{Serial Singularities : Projection in workspace and joint space}

Serial singularities occur when the determinant of the inverse kinematics matrix $\mathbf{B}$ vanishes. When the manipulator is in such a singularity, there is a direction along which no Cartesian velocity can be produced. Serial singularity analysis of delta-like robot is done using the function SerialSingularities() shown in Listing 14.

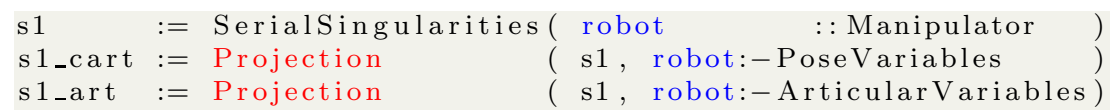

Listing 14: Projection of serial singularities in workspace and joint space

In Eq.(9) $, \operatorname{det}(\mathbf{B})_{o}, \operatorname{det}(\mathbf{B})_{h}, \operatorname{det}(\mathbf{B})_{t}$ and $\operatorname{det}(\mathbf{B})_{u}$ are the serial singularities of Orthoglide, Hybridglide, Triaglide and UraneSX, respectively. One can compute (the algebraic closure of) the projection of the serial singularities in the joint space and workspace. Both are then defined as the zero set of some system of algebraic equations and we assume that the considered robots are generic enough so that both are hypersurfaces. Also these surfaces are shown in Fig.(10) and Fig.(11).

$$
\begin{aligned}
\operatorname{det}(\mathbf{B})_{o} & =\left(\rho_{1}-x\right)\left(\rho_{2}-y\right)\left(\rho_{3}-z\right) \\
\operatorname{det}(\mathbf{B})_{h} & =\left(\rho_{1}-y\right)\left(\rho_{2}-y\right)\left(\rho_{3}-z\right) \\
\operatorname{det}(\mathbf{B})_{u} & =\left(\rho_{1}-z\right)\left(\rho_{2}-z\right)\left(\rho_{3}-z\right) \\
\operatorname{det}(\mathbf{B})_{t} & =\left(\rho_{1}-y\right)\left(\rho_{2}-y\right)\left(\rho_{3}-y\right)
\end{aligned}
$$




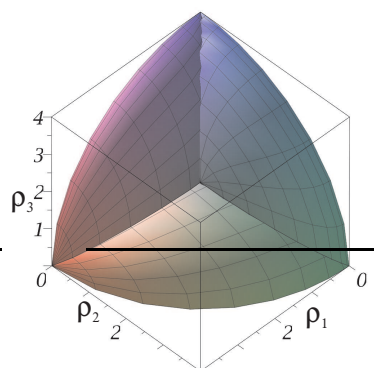

(a)

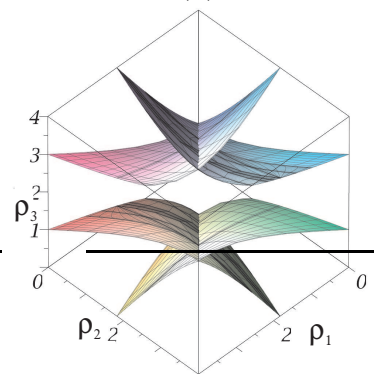

(c)

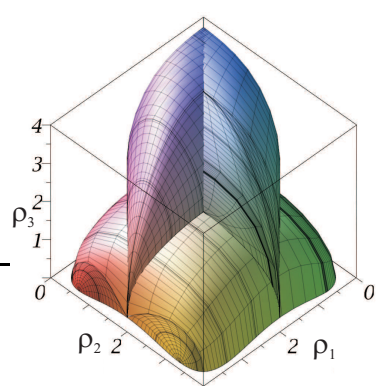

(b)

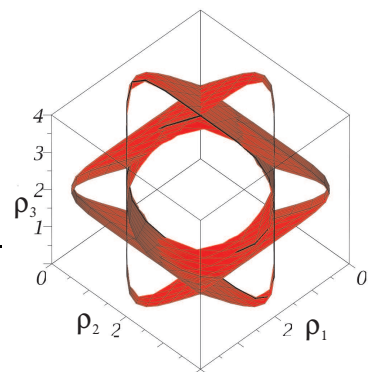

(d)

FIGURE 10: Projection of serial singularity surfaces in the joint space for (a) Orthoglide, (b) Hybridglide, (c) Triaglide and (d) UraneSX

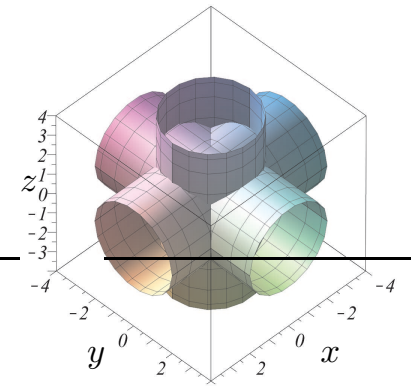

(a)

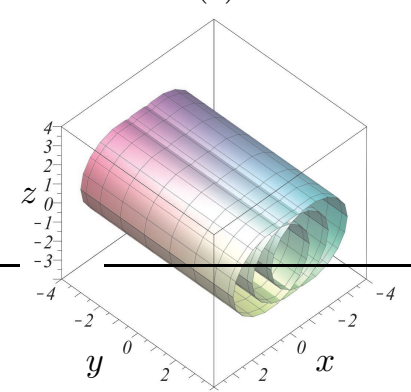

(c)

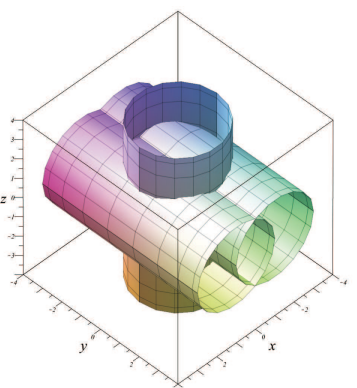

(b)

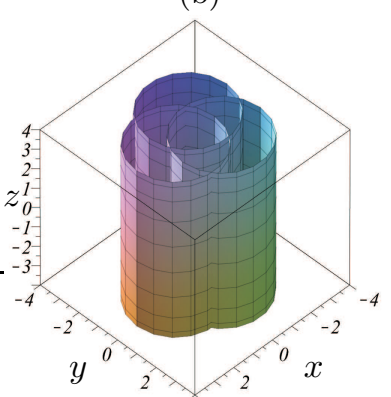

(d)

FIGURE 11: Projection of serial singularity surfaces in the workspace for (a) Orthoglide, (b) Hybridglide, (c) Triaglide and (d) UraneSX 
Figure (10) shows the projections of singularity surface $s 1$ in joint space and $s 1 \_a r t$ is the projection surface. And $s 1_{\text {_ccart }}$ is the projection surface in workspace and is shown in Fig. (11).

\subsection{Complexity in Singularities}

In Table 3and 4, a comparative study of five parameters among the family of delta like robot is presented. We have tabulated the main characteristics of the polynomials (In three variables) used for the plots (Implicit surface) : their total degree, their number of terms and the maximum bitsize of their coefficients. We have also reported the time (In seconds) for plotting the implicit surface which they define and the number of cells computed by the CAD, as well as the number of plotted cells where the equations admits real solutions in the plotted range.

This function is more precise than Maple's "implicitplot3d" function because it calculates all the singular places of the surface and then triangulates it. The surface is not calculated only a discretization of the space. Several functions are used which involves the discriminant variety, Gröbner bases and CAD computations, computed in Maple 2018 with a Intel(R) Core(TM) i7-5600U CPU 2.60 $\mathrm{GHz}$ (16 Gb RAM). As can be seen from Table 3, there exists higher values of all the parameters for the Hybridglide, among all manipulators listed, which infers that it has more complex parallel singularities, whereas for the Triaglide all the values are least which intuits the less complicated singularities. For example, the computation times for the Hybridglyde for parallel singularities is high compared to the one for the Othoglide, even if the surface has similar characteristics. This is due to the geometry of the surface which is more difficult to decompose in the case of the Hybridglide : the CAD is described by 636 cylindrical cells in the case of Hybridglide while it is described by 300 cells for the Orthoglide because a large part of the singularity surface is a sphere.

From Table 4, it can be inferred that there exists higher values of all the parameters for the UraneSX, among all manipulators listed, which infers that it has more complex serial singularities, whereas for the Triaglide all the values are least which intuits the less complicated singularities. The computation times for plotting the serial singularities in joint space is higher compared to others.

\section{Workspace Analysis of a Delta-like Family Robot}

The workspace analysis allows to characterize of the workspace regions where

the number of real solutions for the inverse kinematics is constant. A CAD algorithm is used to compute the workspace of the robot in the projection space $(x, y, z)$ with some joint constraints (shown in Eq. 2) taken in account.

The three main steps involved in the analysis are [17, 26, 34] :

- Computation of a subset of the joint space (resp. workspace) where the number of solutions changes : the Discriminant Variety .

- Description of the complementary of the discriminant variety in connected cells : the Generic CAD.

- Connecting the cells belonging to the same connected component in the counterpart of the discriminant variety : interval comparisons.

${ }_{530}$ Table 5 shows the number of cells corresponding to the number of solutions in the workspace, which is the outcome of cell decomposition. The different 
TABLE 3: Comparison of the different parameters associated with the projection of parallel singularity surface in the workspace and joint space for the delta-like robots

\begin{tabular}{|c|c|c|c|c|c|}
\hline \multicolumn{7}{|c|}{ PARALLEL SINGULARITIES } \\
\hline Panipulators & Plotting Time & Degrees & No.of terms & Binary & No. of Cells \\
\hline Orthoglide & 07.650 & $18[10,10,10]$ & 097 & 015 & {$[0300,42]$} \\
\hline Hybridglide & 23.536 & $20[16,08,12]$ & 119 & 017 & {$[0636,80]$} \\
\hline Triaglide & 13.682 & $03[00,00,03]$ & 002 & 002 & {$[0150,04]$} \\
\hline UraneSX & 25.100 & $06[06,04,00]$ & 015 & 040 & {$[2795,66]$} \\
\hline \multicolumn{7}{|c|}{ Projection in Joint Space } \\
\hline Orthoglide & 00.850 & $06[04,04,04]$ & 010 & 004 & {$[012,01]$} \\
\hline Hybridglide & 02.917 & $06[04,04,04]$ & 025 & 006 & {$[111,20]$} \\
\hline Triaglide & 09.589 & $06[04,04,04]$ & 041 & 006 & {$[126,51]$} \\
\hline UraneSX & 02.300 & $06[04,04,04]$ & 041 & 051 & {$[041,09]$} \\
\hline \hline
\end{tabular}

TABLE 4: Comparison of the different parameters associated with the projection of serial singularity surface in the workspace and joint space for the delta-like robots

\begin{tabular}{|c|c|c|c|c|c|}
\hline \multicolumn{7}{|c|}{ SERIAL SINGULARITIES } \\
\hline Projection in Workspace \\
\hline Manipulators & Plotting Time & Degrees & No.of terms & Binary & No. of Cells \\
\hline Orthoglide & 07.230 & $06[04,04,04]$ & 017 & 006 & {$[016,02]$} \\
\hline Hybridglide & 07.243 & $06[06,02,04]$ & 015 & 006 & {$[045,03]$} \\
\hline Triaglide & 05.437 & $06[06,06,00]$ & 010 & 006 & {$[045,03]$} \\
\hline UraneSX & 10.700 & $06[12,12,00]$ & 013 & 036 & {$[045,03]$} \\
\hline \multicolumn{7}{|c|}{ Projection in Joint Space } \\
\hline Orthoglide & 03.957 & $18[12,12,12]$ & 062 & 012 & {$[021,03]$} \\
\hline Hybridglide & 06.173 & $18[12,12,12]$ & 281 & 017 & {$[158,27]$} \\
\hline Triaglide & 06.794 & $06[06,06,06]$ & 042 & 007 & {$[077,17]$} \\
\hline UraneSX & 07.400 & $12[12,12,12]$ & 252 & 151 & {$[108,29]$} \\
\hline \hline
\end{tabular}

TABLE 5: Definition of the Workspace with CAD

\begin{tabular}{|c|c|c|c|c|c|c|}
\hline \multicolumn{7}{|c|}{ Workspace : Number of cells } \\
\hline Number of solutions & 0 & 1 & 2 & 4 & 8 & Total \\
\hline Orthoglide & 28782 & 1196 & 0 & 0 & 130 & 30108 \\
\hline Hybridglide & 93292 & 4484 & 7228 & 4196 & 1164 & 110364 \\
\hline Triaglide & 27708 & 384 & 464 & 420 & 400 & 29376 \\
\hline UraneSX & 9918 & 236 & 36 & 0 & 0 & 10190 \\
\hline \hline
\end{tabular}

shapes of workspace for the delta-like robots is shown in Fig.(12), where blue, red, yellow and green regions correspond to the one, two, four and eight number of solutions for the IKP. A comparative study is done on the workspace of the family of delta-like manipulator and the results are shown in Fig.(12). All the 


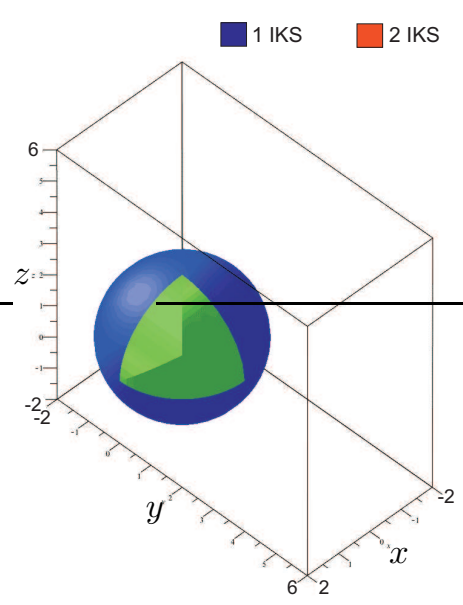

(a)

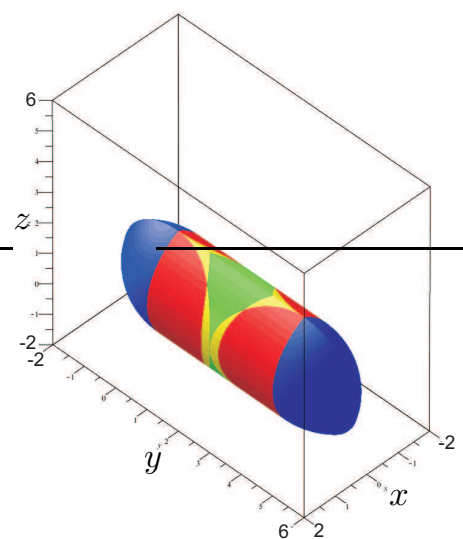

(c)

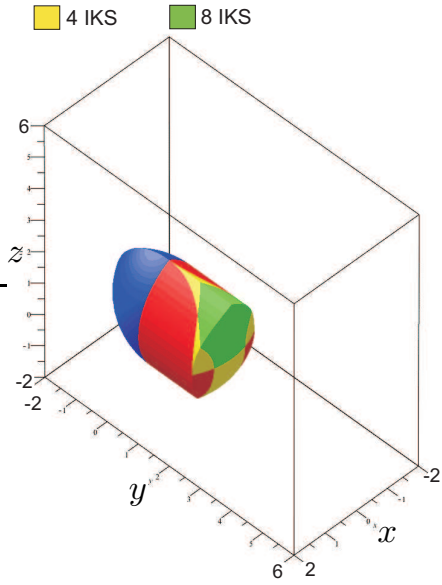

(b)

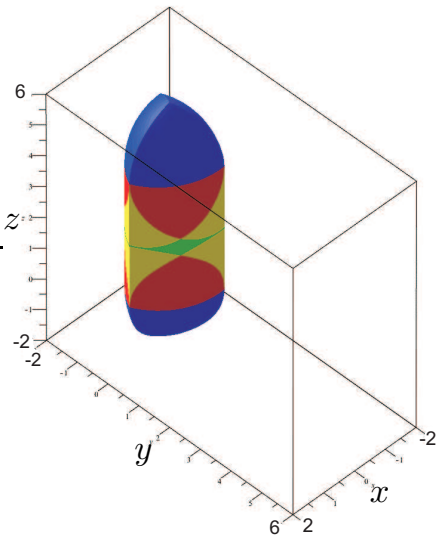

(d)

Figure 12: Workspace plot for Orthoglide (a), Hybridglide (b), Triaglide (c) and UraneSX (d) robot

workspace are plotted in the rectangular box, where $x \in[-2,2], y \in[-2,6]$ and $z \in[-2,6]$, so that the shapes of these workspace can be compared. From the Fig. 12 it can be intuited that the Triaglide will be good selection, if the task space is more in horizontal plane, whereas the Orthoglide is good for the three dimensional task space.

The number of cells in the workspace plays a role in motion generation when one want to know if a path is in the workspace [35]. Indeed, if we discretize the path, a Maple function named "CellLocation" allows us to know where an pose is located from the list of cells calculated with the CAD.

\section{Joint space Analysis of a Delta-like Family Robot}

The Joint space analysis predicts the feasible and non-feasible combinations of the prismatic joint variables which are essential for the parallel robot control. The boundaries of the joint space are either the surfaces associated with the 
parallel singularities or the surfaces associated with the joint limits. The Joint space analysis is done using CAD using the joint limits defined in Eq. 2, These cells are plotted in Fig. (13) where red region corresponds to two solutions for the DKP. One can note that for the Orthoglide and Hybridglide robots, the shape of the joint space is regular and composed of a single connected component. For Triaglide and UraneSX robots, the joint space consists of several components either completely disconnected or connected by single lines. If we want to define a robot with simple joint boundaries (defined by intervals), Orthoglide and Hybridglide robots will be the best selections.

Table 6] shows the number of cells corresponding to the number of solutions in the joint space, which is the outcome of cell decomposition. The number of cells in the joint space plays an important part while simulating robot movements when control or robot geometry errors are introduced into the model. A path that can be achieved in the workspace may be too close to singular configurations after the use of the inverse geometric model including these disturbances [35]. The "CellLocation" Maple function can be used to evaluate the position of the articular trajectory.

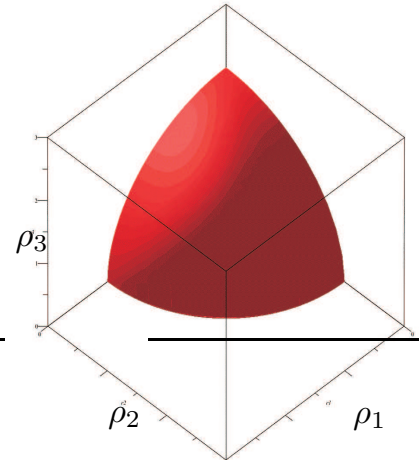

(a)

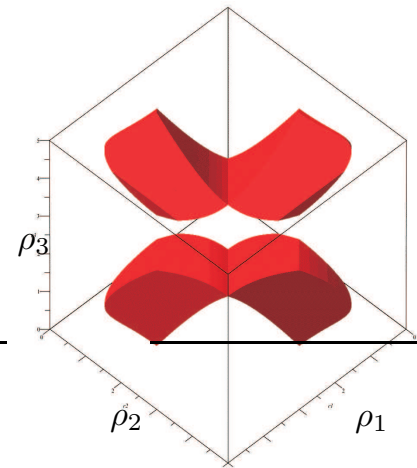

(c)

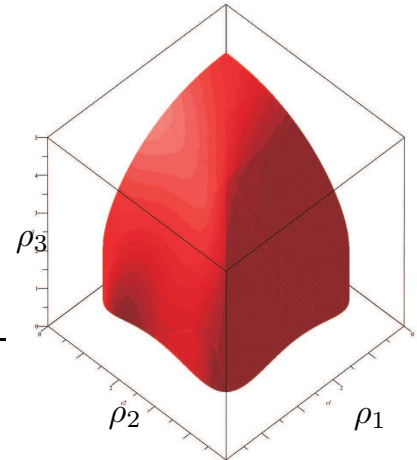

(b)

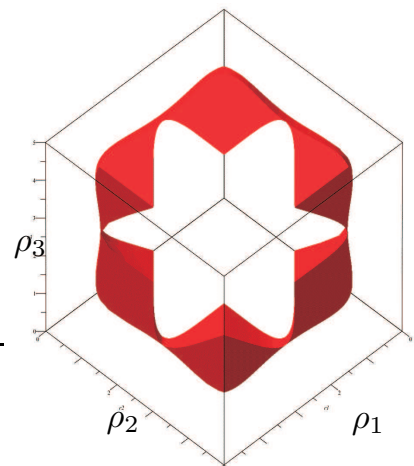

(d)

Figure 13: Joint space plot for Orthoglide (a), Hybridglide (b), Triaglide (c) and UraneSX (d) robot 
TABLE 6: Definition of the Joint space with CAD

\begin{tabular}{|c|c|c|c|c|c|c|}
\hline \multicolumn{7}{|c|}{ Joint space : Number of cells } \\
\hline Number of solutions & 0 & 1 & 2 & 4 & 8 & Total \\
\hline Orthoglide & 10509 & 0 & 4160 & 0 & 0 & 14669 \\
\hline Hybridglide & 98917 & 0 & 3041 & 0 & 0 & 11958 \\
\hline Triaglide & 5375 & 0 & 426 & 0 & 0 & 5801 \\
\hline UraneSX & 50598 & 0 & 4006 & 0 & 0 & 54604 \\
\hline \hline
\end{tabular}

\section{Conclusions}

A comparative study on the workspace of different delta-like robots gives the idea about the shape of the workspace, which further plays an important role in the selection of the manipulator for the specific task or for the trajectory planning. The main characteristics associated with the singularities are tabulated in Table 4and 3, which also gives some information about the complexity of the singularities, which is an essential factor for the singularity-free path plannings. From these data, it can be observed that the singularities associated with the Hybridglide are complicated, whereas the structure of those associated with the Triaglide is rather simple. For the Orthoglide and Hybridglide robots, the shape of the joint space is regular and composed of a single connected component, whereas for Triaglide and UraneSX robots, the joint space consists of several components either completely disconnected or connected by single lines.

[1] Y. Tsai, A. Soni, An algorithm for the workspace of a general nr robot, Journal of Mechanisms, Transmissions, and Automation in Design 105 (1) (1983) 52-57.

[2] J. A. Hansen, K. Gupta, S. Kazerounian, Generation and evaluation of the workspace of a manipulator, The International Journal of Robotics Research 2 (3) (1983) 22-31.

[3] C. Gosselin, Determination of the workspace of 6-dof parallel manipulators, ASME Journal of Mechanical Design 112 (3) (1990) 331-336.

[4] J.-P. Merlet, Geometrical determination of the workspace of a constrained parallel manipulator, Advances in Robot Kinematics, Ferrare, Italy, Sept (1992) 7-9.

[5] G. Castelli, E. Ottaviano, M. Ceccarelli, A fairly general algorithm to evaluate workspace characteristics of serial and parallel manipulators, Mechanics based design of structures and machines 36 (1) (2008) 14-33.

[6] I. A. Bonev, J. Ryu, A new approach to orientation workspace analysis of 6-dof parallel manipulators, Mechanism and machine theory 36 (1) (2001) $15-28$.

[7] D. Chablat, P. Wenger, F. Majou, J.-P. Merlet, An interval analysis based study for the design and the comparison of three-degrees-of-freedom parallel kinematic machines, The International Journal of Robotics Research 23 (6) (2004) 615-624. 
[8] M. Zein, P. Wenger, D. Chablat, An exhaustive study of the workspace topologies of all $3 \mathrm{r}$ orthogonal manipulators with geometric simplifications, Mechanism and Machine Theory 41 (8) (2006) 971-986.

[9] E. Ottaviano, M. Husty, M. Ceccarelli, Identification of the workspace boundary of a general 3-r manipulator, Journal of Mechanical Design 128 (1) (2006) 236-242.

[10] D. Chablat, R. Jha, F. Rouillier, G. Moroz, Non-singular assembly mode changing trajectories in the workspace for the 3-rps parallel robot, in : Advances in Robot Kinematics, Springer, 2014, pp. 149-159.

[11] D. Chablat, G. Moroz, P. Wenger, Uniqueness domains and non singular assembly mode changing trajectories, in : Robotics and Automation (ICRA), 2011 IEEE International Conference on, IEEE, 2011, pp. 3946-3951.

[12] R. Jha, Contributions to the performance analysis of parallel robots, Ph.D. thesis, Ph. D. Thesis, École Centrale de Nantes (2016).

[13] B. Siciliano, O. Khatib, Springer handbook of robotics, Springer, 2016.

[14] J. Snyman, L. Du Plessis, J. Duffy, An optimization approach to the determination of the boundaries of manipulator workspaces, Journal of Mechanical Design 122 (4) (2000) 447-456.

[15] I. A. Bonev, C. M. Gosselin, Analytical determination of the workspace of symmetrical spherical parallel mechanisms, IEEE Transactions on Robotics 22 (5) (2006) 1011-1017.

[16] O. Bohigas, M. Manubens, L. Ros, A complete method for workspace boundary determination on general structure manipulators, IEEE Transactions on Robotics 28 (5) (2012) 993-1006.

[17] D. Chablat, R. Jha, F. Rouillier, G. Moroz, Workspace and joint space analysis of the 3-rps parallel robot, in : ASME 2014 International Design Engineering Technical Conferences and Computers and Information in Engineering Conference, American Society of Mechanical Engineers, 2014, pp. V05AT08A056-V05AT08A056.

[18] R. Jha, D. Chablat, F. Rouillier, G. Moroz, Workspace and singularity analysis of a delta like family robot, in : Robotics and Mechatronics, Springer, 2016, pp. 121-130.

[19] R. Jha, D. Chablat, L. Baron, Influence of design parameters on the singularities and workspace of a 3-rps parallel robot, Transactions of the Canadian Society for Mechanical Engineering 42 (1) (2018) 30-37.

[20] P. Wenger, D. Chablat, Kinetostatic analysis and solution classification of a planar tensegrity mechanism, in : Computational Kinematics, Springer, 2018, pp. 422-431.

[21] G. Moroz, F. Rouiller, D. Chablat, P. Wenger, On the determination of cusp points of 3-rpr parallel manipulators, Mechanism and Machine Theory 45 (11) (2010) 1555-1567. 
[22] M. Manubens, G. Moroz, D. Chablat, P. Wenger, F. Rouillier, Cusp points in the parameter space of degenerate 3-rpr planar parallel manipulators, Journal of mechanisms and robotics 4 (4) (2012) 041003.

[23] G. Moroz, D. Chablat, P. Wenger, F. Rouiller, Cusp points in the parameter space of rpr-2prr parallel manipulators, in : New Trends in Mechanism Science, Springer, 2010, pp. 29-37.

[24] S. Caro, P. Wenger, D. Chablat, Non-singular assembly mode changing trajectories of a 6-dof parallel robot, in : ASME 2012 International Design Engineering Technical Conferences and Computers and Information in Engineering Conference, American Society of Mechanical Engineers, 2012, pp. $1245-1254$

[25] J.-P. Merlet, Parallel robots, Vol. 128, Springer Science \& Business Media, 2006.

[26] D. Lazard, F. Rouillier, Solving parametric polynomial systems, Journal of Symbolic Computation 42 (6) (2007) 636-667.

[27] J. Gerhard, D. Jeffrey, G. Moroz, A package for solving parametric polynomial systems, ACM Communications in Computer Algebra 43 (3/4) (2010) $61-72$.

[28] Maple, Cylindrical Algebraic Decomposition, Vol. Maple User Manual, Toronto : Maplesoft, a division of Waterloo Maple Inc., 2005-2015.

[29] Maple, Cell Decomposition, Vol. Maple User Manual, Toronto : Maplesoft, a division of Waterloo Maple Inc., 2005-2015.

[30] A. Pashkevich, D. Chablat, P. Wenger, Kinematics and workspace analysis of a three-axis parallel manipulator : the orthoglide, Robotica 24 (1) (2006) $39-49$.

[31] M. Hebsacker, T. Treib, O. Zirn, M. Honegger, Hexaglide 6 dof and triaglide 3 dof parallel manipulators, in : Parallel kinematic machines, Springer, 1999, pp. 345-355.

[32] D. A. Cox, J. Little, D. O'shea, Using algebraic geometry, Vol. 185, Springer Science \& Business Media, 2006.

[33] G. Moroz, Properness defects of projection and minimal discriminant variety, Journal of Symbolic Computation 46 (10) (2011) 1139-1157.

[34] G. Moroz, Sur la décomposition réelle et algébrique des systemes dépendant de parametres, Ph.D. thesis, Université Pierre et Marie Curie-Paris VI (2008).

[35] R. Jha, D. Chablat, F. Rouillier, G. Moroz, An algebraic method to check the singularity-free paths for parallel robots, in : ASME 2015 International Design Engineering Technical Conferences and Computers and Information in Engineering Conference, American Society of Mechanical Engineers, 2015, pp. V05CT08A002-V05CT08A002. 